\title{
Changes in Polyphenol Content in Raspberry by Cultivation Environment
}

\author{
Ryo Kobori ${ }^{1}$, Seiya Hashimoto ${ }^{1}$, Hayato Koshimizu ${ }^{1}$, Takashi KAWASAKI ${ }^{2}$, and Akiko SAITO ${ }^{*}$ \\ ${ }^{1}$ Advanced Science \& Engineering, Graduate School of Engineering, Osaka Electro-Communication University (OECU), 18-8 \\ Hatsu-cho, Neyagawa-shi, Osaka 572-8530, Japan \\ ${ }^{2}$ Research Institute for Sustainable Humanosphere, Kyoto University, Uji, Kyoto 611-0011, Japan
}

\begin{abstract}
It is known that red raspberry (Rubus idaeus) contains various polyphenol compounds at high concentration and its functionality is expected to have health maintenance and disease prevention effect. Not only the fruits, but also the leaves of raspberry plants, are highly esteemed for tea making around the world and are largely used for food. Cultivation of raspberry in a plant factory unit and analysis of leaf extract under different cultivation conditions such as light intensity, wavelength and temperature showed that flavan-3-ol derivatives increase under blue light confirmed.
\end{abstract}

\section{Introduction}

There is currently great interest in the investigation of compounds from food sources with biological activities, as they are generally considered highly safe because they are consumed as part of the general daily diet. In particular, polyphenols contained in many health foods, as well as in vegetables and fruits, are thought to have various health benefits (Harborne, 1993; Harborne and Baxter, 1999). Among them, flavan-3-ol derivatives are general plantderived physiologically active compounds known to be highly functional. In particular, the main ingredient of green tea-polyphenols shows various moderate bioactivities without severe toxicity and its health promoting effects have been extensively studied. Although various biologically active flavan-3-ol derivatives are present as minor constituents in plants as well as in green tea, their biological activities have yet to be revealed, mainly owing to their relative unavailability. Therefore, the development of new methods to elucidate the biosynthesis pathway, polymerization mechanisms, and transport of flavan-3-ol derivatives has become an important and active field of research (Zhao et al., 2010; Liu et al., 2013). Especially, developing a method to control the biosynthesis of flavan-3-ol derivatives with strong biological activities in plants would be a tool of great value to produce highly functional plants and foods. flavan-3-ol derivatives. For example, short-term ultraviolet $\mathrm{C}$ irradiation was shown to stimulate biosynthetic pathways, leading to enhancement of the accumulation of polyphenol compounds (Wen et al., 2015). Similarly, long-term irradiation of visible blue light showed a similar effect on the amounts of flavonoids produced in Chinese cabbage (Kim et al., 2015). These reports demonstrate the possibility of controlling the amounts of specific polyphenol compounds in plants, including flavan-3-ol derivatives; furthermore, they indicate that environmental conditions has a large influence on polyphenol production, which causes difficulty in the functional evaluation of plant extracts. Flavan-3-ol derivatives are thought to promote health because of their various functionalities. Our next challenge was to achieve the effective, selective production of high-functional flavan-3-ol derivatives in plants. Thus, we selected red raspberry (Rubus idaeus L.) as our experimental model plant because it is a species widely known for its edible fruits and as polyphenol rich and highly beneficial for human health (Hummer, 2010; Rao and Synder, 2010). Furthermore, they are most commonly known as food products and their extracts are also known as anti-inflammatory (Szymanowska et al., 2018) and antimicrobial properties (Olle et al., 2012). As mentioned above, numerous studies report increasing polyphenol content in various plants by light irradiation management. Therefore, we planned studies to irradiate raspberries with LED light to increase polyphenols, especially flavan-3-ol compounds. In this report, we

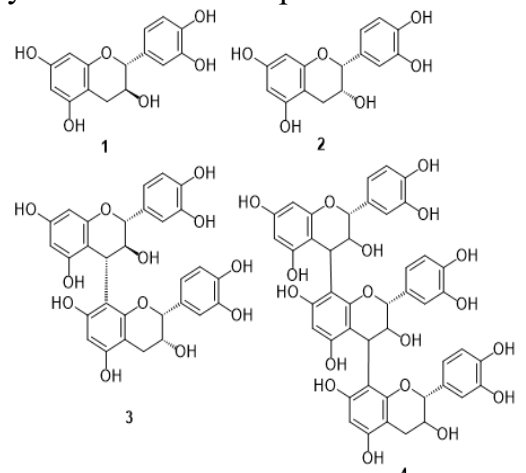

Figure 1. The structures of flavan-3-ol derivatives contained in raspberry

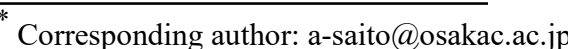


discuss the method for raspberry cultivation in a plant factory unit kept under constant environmental conditions, and the response in polyphenol production in the leaves to light wavelength. When blue LED light was used for cultivation, the amounts of total polyphenols and flavan3-ol derivatives (Figure 1), (+)-catechin (1), (-)epicatechin (2), procyanidin B4 (3), and flavan-3-ol trimer (4), were increased. The results of PCR analysis to clarify the reason for the increase suggested that the expression level of polyphenol biosynthesis related gene CHS was increased in the leaves grown with blue light irradiation.

\section{Material and Methods}

Cultivation of raspberry was carried in the plant factory unit, 'Agri cube'. Custom order LED light units that can control light quantity and irradiation wavelength were used for light control within the plant factory unit. Plants were kept under an 18/6 h light dark regime for the duration of the experiment. Leaves were sampled seven days after combined light-temperature treatment initiation.

\subsection{Preparation of crude extract solution of raspberry leaves}

Raspberry leaves were randomly harvested and dried at room temperature for 48 hours. Dried leaves were powdered using a Speed mill MS-05. To suppress heat generation, crushing was carried out three times for $20 \mathrm{~s}$ at $10 \mathrm{~s}$ intervals $(60$ seconds in total). Leaf samples (1.0 g) were immersed in $40 \mathrm{~mL}$ of HPLC grade methanol and extracted for 24 hours at $25^{\circ} \mathrm{C}$. Filtration was carried out using celite as a filtration auxiliary, the filtrate was then concentrated by evaporation under reduced pressure and dried in vacuo. The obtained solid was made into a solution of $100 \mathrm{mg} / \mathrm{mL}$ with DMSO. The DMSO-solution extracts were stored at $-40^{\circ} \mathrm{C}$ until used for biological assay and analysis.

\subsection{Chemical analysis and measurement of radical scavenging activity}

Total phenolic content of the extract was determined by Folin-Ciocalteu method (Folin and Denis, 1915, Juilcunen-Tiittoo, 1985). Proanthocyanidin concentration was determined by a modified protein-precipitation method described by Harbertrson et al. (2002) and Cáceres-Mella et al. (2013). Radical scavenging activity in raspberry extracts was determined by the DPPH $(1,1-$ diphenyl-2-picryl-hydrazyl) (Nanjo et al., 1996; Re et al., 1999). LC-MS analysis conditions. Measurement was performed LC-ESI-MS. Briefly, 0.05\% (v/v) formic acid

was mobile phase A and acetonitrile was mobile phase B. Analyses of raspberry extracts were achieved using a linear gradient from $0-35 \% \mathrm{~B}$ over $0-90 \mathrm{~min} ; 35-100 \% \mathrm{~B}$ over 90-95 min; $100 \% \mathrm{~B}$, over $95-100 \mathrm{~min} ; 0 \% \mathrm{~B}$, over $100-110 \mathrm{~min}$. The flow rate was set at $0.2 \mathrm{ml} / \mathrm{min}$. The injection volume was $10 \mu \mathrm{l}$ and the temperature of the column oven was maintained at $40{ }^{\circ} \mathrm{C}$.

\subsection{Raspberry cultivation in a plant factory unit and LED light irradiation}

Table 1 shows the raspberry cultivation conditions in the plant factory unit and the dry weight of the methanol extracts obtained from $1.0 \mathrm{~g}$ samples of dried leaves. Conditions $\mathrm{A}$ and $\mathrm{C}$ consisted in commercially available white LED light; conditions B, D, E, F, G and I varied by changing the proportion of blue and red LED lights. Cultivation under condition $\mathrm{H}$ consisted in no LED light provision. Each cultivation condition was maintained for 7 days, after which the leaves were harvested for extraction. Cultivation conditions did not largely affect the extracted weight obtained; however, when plants were cultivated under blue light only, the extract tended to be less. Each extract was adjusted to $100 \mathrm{mg} / \mathrm{mL}$ solution with dimethyl sulfoxide (DMSO) and used for quantitative analysis and radical scavenging activity.

\section{Results and Discussion}

\subsection{Analysis of total polyphenols and total proanthocyanidin amounts in extracts from raspberry leaves}

Figure 2 shows the total polyphenol content measured by the Folin-Ciocalteu method and the total proanthocyanidin content using BSA adsorption. When grown with white LED light (conditions A and C), both, total polyphenols and total proanthocyanidins decreased at $18{ }^{\circ} \mathrm{C}$, confirming that temperature had a significant effect on polyphenol production. In the case of cultivation with only blue LED light (conditions B and D), the effect of temperature on total polyphenol amount was small, and total proanthocyanidin was increased at $22^{\circ} \mathrm{C}$. In addition, under the condition $\mathrm{G}$ in which the blue LED light was intensified, the total polyphenol amount slightly increased. The amount of flavan-3-ol derivatives suggested that temperature effect was small under blue LED light. When red and blue LED lights were used at the same time (conditions E, F and I), total polyphenol content of condition I increased greatly.

\subsection{Radical scavenging activity}

Table 1. Cultivation conditions and amount of extract oldtained from raspberry leaves

${ }^{1}$ In conditions A to B, cultivation was carried out using commercially available white LED. ${ }^{2}$ Dry weight of the extract pbtained from $1.0 \mathrm{~g}$ samples of dried leaves cultivated under each condition.

\begin{tabular}{|c|c|c|c|c|c|c|c|c|c|}
\hline & $\mathbf{A}^{1}$ & $\mathbf{B}^{1}$ & $\mathbf{C}$ & D & $\mathbf{E}$ & $\mathbf{F}$ & $\mathbf{G}$ & $\mathbf{H}$ & $\mathbf{I}$ \\
\hline PPFD $\left(\mu \mathrm{mol} \mathrm{m} \mathrm{m}^{-2} \mathrm{~s}^{-1}\right)$ Blue & 7.23 & 30.24 & 7.23 & 30.24 & 3.86 & 27.50 & 40.09 & - & 16.54 \\
\hline PPFD $\left(\mu \mathrm{mol} \mathrm{m} \mathrm{m}^{-2} \mathrm{~s}^{-1}\right) \mathrm{Red}$ & 8.33 & - & 8.33 & - & 4.43 & 29.84 & - & - & 27.87 \\
\hline Temperature $\left({ }^{\circ} \mathrm{C}\right)$ & 18 & 18 & 22 & 22 & 22 & 22 & 22 & 22 & 22 \\
\hline Extract $(g)^{2}$ & 0.37 & 0.32 & 0.30 & 0.28 & 0.30 & 0.37 & 0.25 & 0.30 & 0.33 \\
\hline
\end{tabular}




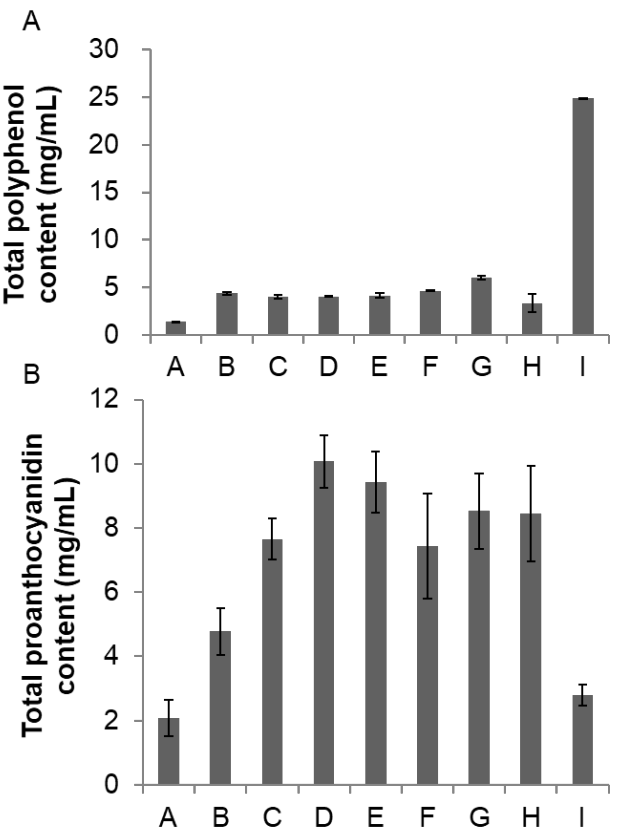

Figure 2. Total polyphenol content and proanthocyanidin content of raspberry leaves cultivated under the different LED light treatment. (a) Total polyphenols were determined by the Folin-Ciocalteu method. Data are mg of gallic acid equivalent per $100 \mathrm{mg} / \mathrm{mL}$ DMSO solution of raspberry leaf extract; (b) Total proanthocyanidin was determined by a modified protein precipitation method. Data are $\mathrm{mg}$ of procyanidin B2 equivalent per 100 $\mathrm{mg} / \mathrm{mL}$ DMSO solution of raspberry leaf extract. Error bars represent SD of the mean $(n=4)$

The radical scavenging activity assay is commonly used as an index of antioxidant activity. As shown in Figure 3, the radical scavenging activity of raspberry extracts was estimated by the DPPH radical scavenging property. Raspberry leaves grown with commercial white LED (conditions $\mathrm{A}$ and $\mathrm{C}$ ), both radical scavenging activities were reduced at $18{ }^{\circ} \mathrm{C}$. This result correlated with total polyphenols and total proanthocyanidin. When only the blue LED was irradiated (conditions B, D and G), there was no large difference due to the cultivation temperature, and the activity increased by the light amount.

\subsection{HPLC analysis and identification of polyphenols contained in raspberry leaf extracts}

Next, identification of the polyphenol compounds in the extracts was carried out by estimation of molecular weight by LCMS analysis and comparison with standards (McCallum et al., 2010; Krauze-Baranowska et al., 2014). In addition to the flavan-3-ol derivatives shown in Figure 1, 1-galloylpedunculagin (5), sanguiin H-6 (6) and

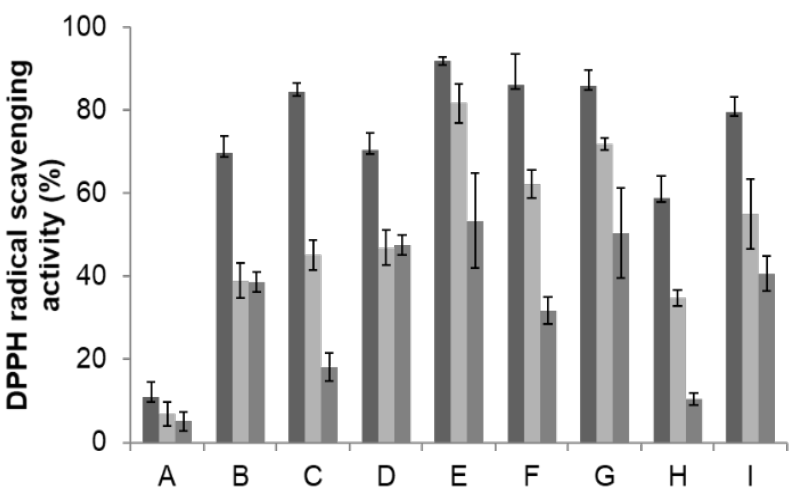

Figure 3. Radical scavenging activity in raspberry leaf extracts. DPPH radical scavenging activity; one $\mathrm{mL}$ of $30 \mu \mathrm{M}$ DPPH solution in EtOH was added to $1 \mu \mathrm{L}$ of raspberry leaf extract in DMSO solution for a final concentration of 1,5 , or 10 $\mu \mathrm{g} / \mathrm{mL}$ (dark gray bar, pale gray bar and gray bar, respectively). Vitamin $\mathrm{E}$ was used as a control. Error bars represent standard deviation of the mean $(\mathrm{n}=8)$

lambertianin C (7) were identified. The HPLC chromatograms of each of the raspberry extracts grown under conditions $\mathrm{C}$ to I are shown in Figure 4.

Cultivation under blue light (condition D and G) promoted the accumulation of flavan-3-ol derivatives (compounds 1 to 4), but reduced the hydrolyzable tannins (compounds 5 to 7). In particular, under condition D, the relative content of flavan-3-ol derivatives (compounds $\mathbf{1}$ to 4) was significantly increased. Cultivation of red raspberry under blue LED light has been suggested to be able to efficiently increase flavan-3-ol derivatives. On the other hand, when red light and blue light were simultaneously irradiated, hydrolysable tannin increased. This suggests that red light irradiation may increase the content of hydrolyzable tannins (compounds 5 to 7). Surprisingly, in the condition $\mathrm{H}$ without light irradiation, the content of flavan-3-ol derivative was relatively increased, and the amount of hydrolyzable tannin was significantly reduced. 

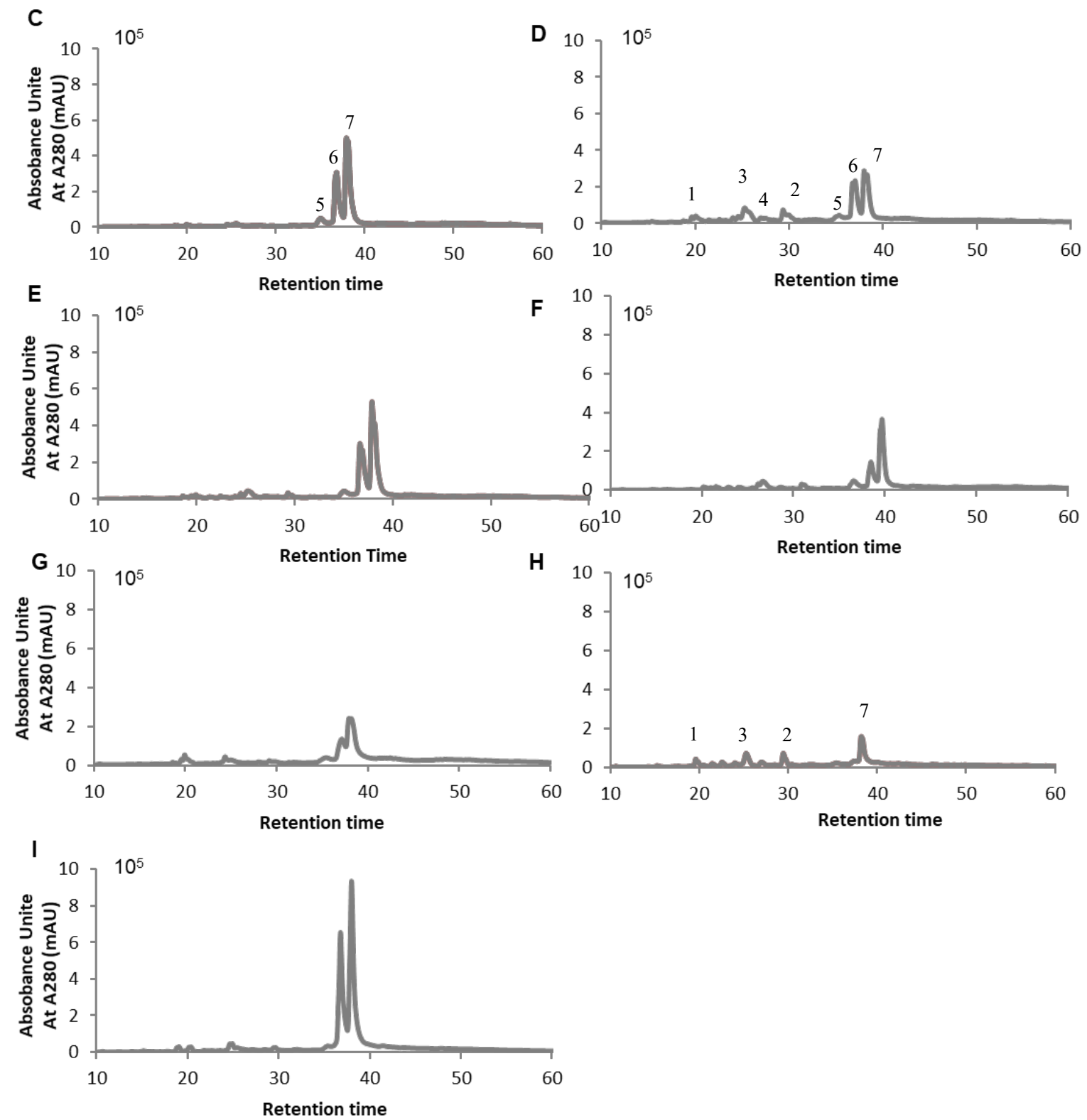

Figure 4. HPLC chromatograms of leaf extracts obtained from raspberry plants cultivated under condition A to $H$. Peak numbers are: 1: (+)-catechin; 2: (-)-epicatechin; 3: procyanidin B4; 4: flavan-3-ol trimer; 5: 1galloylpedunculagin; 6: sanguiin $\mathrm{H}-6$; 7: lambertianin $\mathrm{C}$

\subsection{Semi-quantitative RT-PCR analysis of expression level of the flavan-3-ol derivative biosynthetic enzyme}

As it was suggested that polyphenol content might change depending on the light wavelength and temperature, we next confirmed the influence of cultivation environment on polyphenol biosynthetic enzymes (Kim et al., 2015; MaCallum et al., 2010; Kassaim et al., 2009). From the HPLC measurement result of Figure 4, blue light irradiation increased the amount of procyanidin B 4 in the flavan-3-ol derivative. This compound is a dimer of flavan-3-ol, and in order to increase it, it is necessary to increase the amount of leucocyanidin which is its precursor. Therefore, experiments were performed with an expectation that the expression level of DFR that synthesizes leucocyanidin may be increased, or the expression level of ANS may be decreased.

Figure 5 shows the semi-quantitative RT-PCR analysis of expression level of the flavan-3-ol derivative biosynthetic genes, $C H S, F 3$ ' $H, F L S, D F R$, and $A N S A s$ a result, it was suggested that there was almost no difference in the expression levels of the five genes examined at any temperature. As for the wavelength of light, it was confirmed that CHS is expressed in a large amount under the condition $\mathrm{D}$ grown under blue light irradiation. Further experiments are currently underway to understand why changes in polyphenol compound production occur due to the wavelength and intensity of the irradiated light. 


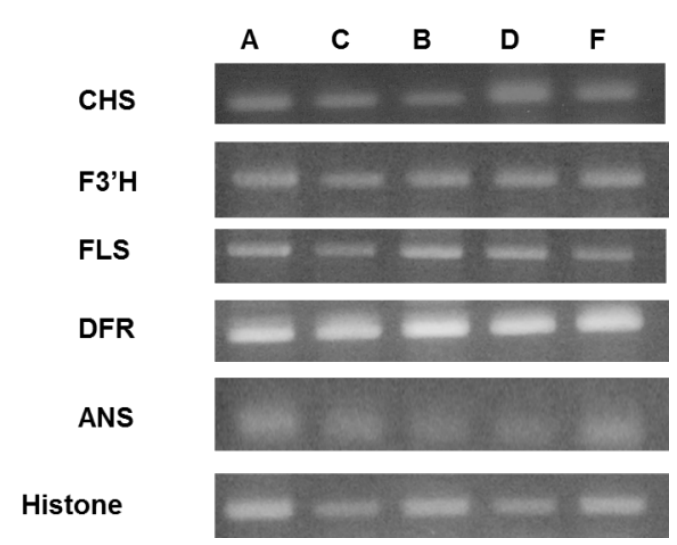

Figure 5. Expression level of flavan-3-ol biosynthesis enzyme encoding genes in raspberry leaves under conditions A, B, C, D, and F. Total RNA was extracted from fresh leaves after 7 days LED light irradiation treatment and used for sqRT-PCR analysis

\section{Conclusions}

In order to clarify the influence of cultivation conditions such as the wavelength and temperature of light to be irradiated on the polyphenol content and flavan-3-ol content of raspberry leaves, studies were conducted using samples grown under a constant environment. As a result of HPLC analysis, it has been suggested that blue light irradiation may increase the content of flavan-3-ol derivative.

\section{Acknowledgements}

I heartedly acknowledge researchers at the Central Research Laboratory, Daiwa House Industry Co., Ltd., for their most helpful advice on raspberry cultivation within the plant factory unit. Similarly, I wish to express my sincere gratitude to the researchers at the Toyama Prefectural Agricultural, Forestry \& Fisheries Research Center, who instructed me on the basic principles of raspberry cultivation.

\section{References}

Cáceres-Mella, Á., Á. Peña-Neira, J. Narváez-Bastias, C. Jara-Campos, R. López-Solís, and J. M. Canals; "Comparison of Analytical Methods for Measuring Proanthocyanidins in Wines and their Relationship with Perceived Astringency," Int. J. Food Sci. Technol., 48, 2588-259 (2013)

Folin, O.; Denis, W.; "A Colorimetric Method for the Determination of Phenols (and Phenol Derivatives) in Urine," J. Biol. Chem., 22, 305-308 (1915)

Harborne, J. B.; The Flavonoids: Advances in Research from 1986, Chapman and Hall London, U.K. (1993)
Harborne, J. B. and H. Baxter; The Handbook of Natural Flavonoids, Wiley, New York, U.S.A. (1999)

Harbertrson, J. F., J. A. Kennedy, and D. O. Adams; "Tannin in Skins and Seeds of Cabernet Sauvignon, Syrah, and Pinot noir Berries during Ripening," Am. J. Enol. Vitic., 53, 54-59 (2002)

Hummer, K. E., "Rubus Pharmacology: Antiquity to the Present," HortScience, 45, 1587-1591 (2010)

Juilcunen-Tiittoo, R.; "Phenolic Constituents in the Leaves of Northern Willows: Methods for the Analysis of Certain Phenolics," J, Agric. Food Chem., 33, 213-217 (1985)

Kassaim, A., J. Poette, A. Paterson, D. Zait, S. McCallum, M. Woodhead, K. Snith,; C. Hackett, and J. Graham; "Environmental and Seasonal Influences on Red Raspberry Anthocyanin Antioxidant Contents and Identification of Quantitative Traits Loci (QTL)," Mol. Nutr. Food Res., 53, 625-624 (2009)

Kim, Y. J., Y. B. Kim, X. Li, S. R. Choi, S. Park, J. S. Park,; Y. P. Lim, and S. U. Park; "Accumulation of Phenylpropanoids by White, Blue, and Red Light Irradiation and Their Organ-Specific Distribution in Chinese Cabbage (Brassica rapa ssp. pekinensis)," J. Agric. Food Chem., 63, 6772-6778 (2015)

Krauze-Baranowska, M., D. Glod, M. Kula, M. Majdam, R. Halasa, A. Matkowski, W. Kozlowska, and A. Kawiak; "Chemical Composition and Biological Activity of Rubus Idaeus Shoots-a Traditional Herbal Remedy of Eastern Europe," BMC Compl. Altern. Med., 14, 480 (2014)

Liu, Y., Z. Shi, S. Maximova, M. J. Payne, and M. J. Guiltinan; "The Mysteries of Proanthocyanidin Transport and PolymerizationProanthocyanidin Synthesis in Theobroma Cacao: Genes Encoding Anthocyanidin Synthase, Anthocyanidin Reductase, and Leucoanthocyanidin Reductase," BMC Plant Biol., 13, 202 (2013)

McCallum, S., M. Woodhead, C. A. Hackett, A. Kassim, and A. Paterson; "Genetic and Environmental Effects Influencing Fruit Colour and QTL Analysis in Raspberry," Theor. Appl. Genet., 121, 611-627 (2010)

Nanjo, F., K. Goto, R. Seto, M. Suzuki, M. Sakai, and Y. Hara,; "Scavenging Effects of Tea Catechins and Their Derivatives on 1,1-diphenyl-2-picrylhydrazyl Radical," Free Radic. Biol. Med., 21, 895-902 (1996)

Olle, M.; Ngouajio, M.; Siomos, A; "Vegetable quality and productivity as influenced by growing medium," Žemdirbyste Agriculture., 99, 399-408 (2012)

Rao, A. V. and D. M. Synder; "Raspberries and Human Health: A Review," J. Agri. Food Chem., 58, 3871-3883 (2010) 
Re, R., N. Pellegrini, A. Proteggente, A. Pannala, and M. Yang; "Antioxidant Activity Applying an Improved ABTS Radical Cation Decolorization Assay," Free Rad. Biol., Med., 26, 1231-1237 (1999)

Szymanowska, U., B. Baraniak, and A. Bogucka-Kocka; "Antioxidant, Anti-Inflammatory, and Postulated Cytotoxic Activity of Phenolic and Anthocyanin-Rich Fractions from Polana Raspberry (Rubus idaeus L.) Fruit and Juice-In Vitro Study," Molecules, 23, E1812 (2018) Wen, P. F., W. Ji, M. Y. Gao, T. Q. Niu, Y. F. Xing, and $\mathrm{X}$. Y. Niu; "Accumulation of flavanols and expression of leucoanthocyanidin reductase induced by postharvest UV-C irradiation in grape berry," Genet Mol Res., 14, 7687-7695 (2015)

Zhao, J., Y. Pang, and R. A. Dixon; "The Mysteries of Proanthocyanidin Transport and Polymerization," Plant Physiol., 153, 437-443 (2010) 